\title{
Trabectedin in Advanced Sarcomas-Experience at a Tertiary Care Center and Review of Literature
}

\author{
Saurav Verma ${ }^{1} \quad$ Kaushal Kalra $^{1} \quad$ Sameer Rastogi ${ }^{1} \quad$ Ekta Dhamija $^{2} \quad$ Avinash Upadhyay $^{1} \quad$ Abhenil Mittal $^{1}$ \\ Aditi Aggarwal $^{3}$ Shamim Ahmed Shamim
}

${ }^{1}$ Department of Medical Oncology, Institute Rotary Cancer Hospital, All India Institute of Medical Sciences, New Delhi, India

2Department of Radiology, Institute Rotary Cancer Hospital, All India Institute of Medical Sciences, New Delhi, India

${ }^{3}$ Department of Radiotherapy, National Cancer Institute, Haryana, Jhajjar, India

${ }^{4}$ Department of Nuclear Medicine, All India Institute of Medical Sciences, New Delhi, India

Address for correspondence Sameer Rastogi, MD, DM, Department of Medical Oncology, Dr. BRA IRCH, AlIMS, Room no. 216, $2^{\text {nd }}$ floor, Ansari Nagar, New Delhi,India 110029,

(e-mail: samdoc_mamc@yahoo.com).

\section{South Asian J Cancer 2021;10:53-57.}

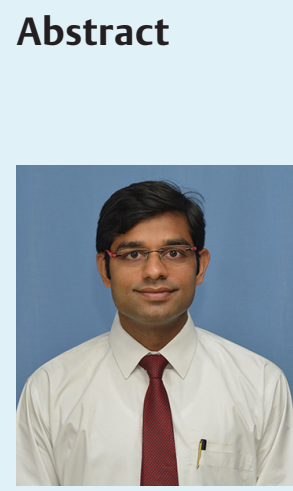

Dr. Sameer Rastogi

\section{Keywords \\ - advanced sarcoma \\ - elastomeric pump \\ - L-sarcomas \\ - leiomyosarcoma \\ - trabectedin}

Background There is sparse literature on trabectedin in advanced soft-tissue sarcomas from developing world. It would be interesting to know about use and outcomes of trabectedin in Indian patients.

Method In a retrospective study, consecutive patients treated with trabectedin from 2016 to 2019 were analyzed. Patients with L-sarcomas were treated at a dose of $1.5 \mathrm{mg} / \mathrm{m}^{2}$, while those with translocation-related sarcomas were treated at a dose of $1.2 \mathrm{mg} / \mathrm{m}^{2}$ as a 24-hour infusion through peripherally inserted central catheter line. From July 2019, infusions were administered through an ambulatory elastomeric pump, while before that patients were admitted for 24 hours. We used SPSS version 23.0 for statistical calculation.

Result A total of 20 patients received trabectedin with a total of 116 infusions. The median age was 46 years (range: $22-73$ years). The male $(n=11,55 \%)$ and female patients were almost equal $(n=9,45 \%)$. Thirteen patients $(65 \%)$ had Eastern Cooperative Oncology Group Performance Status 1. Majority of the patients had leiomyosarcoma ( $n=8,40 \%)$; remaining comprised of liposarcoma (3,15\%), translocation-related sarcomas excluding myxoid liposarcoma $(n=8,40 \%)$ and others $(n=1,5 \%)$. Most common site was extremity $(n=11,55 \%)$ followed by retroperitoneal $(n=3,15 \%)$, visceral ( $n=3,15 \%)$, and others $(n=3,15 \%)$. Median number of previous lines received was 2 (range: $0-4)$. Median number of trabectedin cycles received was 4 (range: $1-17)$. Best response assessed was stable disease $(n=10,50 \%)$, progressive disease $(n=6,30 \%)$, partial response $(n=1,5 \%)$, and not assessed in 3 patients. After a median follow-up of 19 months, median progression-free survival was 4 months.

Conclusion In this heavily treated population (composed of L-sarcomas and translocation-related sarcomas) with many patients with poor performance status, the outcome with trabectedin is in synchrony with literature. However, the need of 24-hour admission might deter quality of life. Elastomeric pump seems to be a reasonable alternative to admission and can be a breakthrough in administering trabectedin, especially in developing countries.
DOI https://doi.org/10.1055/s-0041-1734336 ISSN 2278-330X

How to cite this article: Verma $S$, Kalra K, Rastogi $S$, Dhamija E, Upadhyay A, Mittal A, Aggarwal A, Shamim S.A. Trabectedin in Advanced Sarcomas-Experience at a Tertiary Care Center and Review of Literature South Asian J Cancer 2021;10(2):53-57.
(C) 2021. MedIntel Services Pvt Ltd.

This is an open access article published by Thieme under the terms of the Creative Commons Attribution-NonDerivative-NonCommercial-License, permitting copying and reproduction so long as the original work is given appropriate credit. Contents may not be used for commercial purposes, or adapted, remixed, transformed or built upon. (https://creativecommons.org/licenses/by-nc-nd/4.0/).

Thieme Medical and Scientific Publishers Private Ltd A-12, Second Floor, Sector -2, NOIDA -201301, India 


\section{Introduction}

Soft tissue sarcomas are rare and extremely heterogeneous group of neoplasms arising from mesenchymal tissues. ${ }^{1}$ Treatment of advanced soft tissue sarcomas has evolved in last decade with inclusion of newer therapies like pazopanib, trabectedin, and eribulin apart from conventional chemotherapeutic agents. ${ }^{2,3}$ Trabectedin is a marine derivative that was initially derived from Caribbean sea sponge Ecteinascidia turbinata and causes DNA damage by binding to minor groove of DNA. ${ }^{4}$ It has pleiotropic actions leading to altered cell cycle regulation, transcription factor inhibition, antiangiogenesis, apoptosis, and changes in tumor microenvironment.-5.7t was initially approved by European Medicines Agency based upon phase 2 randomized trials comparing 3 weekly and weekly schedule (3.3 vs. 2.3 months). ${ }^{8-11}$ Subsequently in a trial by Demetri et al trabectedin was compared with dacarbazine and showed improved progression free survival (PFS; 4.2 vs. 1.5 months) in advanced L-sarcomas previously treated with at least two lines of therapy. ${ }^{12}$ This lead to U.S. Food and Drug Administration approval of trabectedin in October 2015. However, this trial was criticized for not meeting its primary end-point and conspicuous absence of evaluation of quality of life (QoL). ${ }^{13}$ Subsequently, based upon its mechanism of action it was tried in translocation-related sarcomas in various reports. ${ }^{14}$

Kawai et al in a phase 2 randomized trial compared trabectedin with best supportive care in patients $(n=73)$ with translocation-related sarcoma in patients who had received multiple lines of treatment. There was significant and very relevant difference of 4.7 months PFS between the two arms. ${ }^{15}$ A subset analysis analyzing mesenchymal chondrosarcoma and extraskeletal myxoid chondrosarcoma showed staggering difference of 12.5 versus 1 month showing its potential effectiveness in this population. ${ }^{16}$

In the current report, we have analyzed our single-center experience of trabectedin in L- sarcomas and translocation-related sarcomas.

\section{Materials and Methods}

This was a single institution retrospective study where all advanced L-sarcoma and translocation-related sarcoma patients treated with trabectedin between January 2016 and December 2019 were included using the hospital-based data system. Toxicity and outcome data were retrieved from a well-structured proforma.

\section{Treatment Characteristics}

Patients with leiomyosarcoma and liposarcoma were treated at a dose of $1.5 \mathrm{mg} / \mathrm{m}^{2}$ while those with translocation-related sarcomas at $1.2 \mathrm{mg} / \mathrm{m}^{2}$ as 24-hour infusion through peripherally inserted central catheter [PICC] line or chemo-port. Since July 2019, infusions were administered through an elastomeric pump while previous to that patients were admitted for 24 hours for administration via PICC line. Patients were followed up initially after a week and then every 3 weeks, with clinical examination focusing on toxicity, disease control, and laboratory investigation including complete hemogram, liver and renal function tests, and creatine phosphokinase.

\section{Data Collection and Toxicity Grading}

Demographic details (including age, sex, Eastern Cooperative Oncology Group performance status [ECOG PS]), diagnosis, and various treatment lines received by the patient before trabectedin were documented. Treatment response and present status of the patients were obtained telephonically and from records. Toxicity was graded using common terminology criteria for adverse events version v.4.03. Toxicity data, treatment interruptions, dose reduction, and dose increments were also tabulated.

Categorical variables and ordinal variables were displayed in tabular form. All numerical variables were tabulated as whole numbers. Median and range were used to describe quantitative variables. Kaplan-Meier curve was used for survival analyses. $p$-Values of $<0.05$ were considered statistically significant. IBM SPSS version 23.0. was used for statistical analysis (Armonk, New York, United States).

\section{Results}

\section{Study Population}

During the study period, a total of 20 patients received trabectedin (patient characteristics are mentioned in - Table 1 ). The median age was 46 years (range: $22-73$ years) with a male predominance (55\%). Thirteen patients (65\%) had ECOG PS of 1 at the time of trabectedin initiation; five patients (25\%) had PS 2, and two patients (10\%) had PS 3. Majority of the patients had leiomyosarcoma $(n=8,40 \%)$ and remaining comprised of liposarcoma $(3,15 \%)$, translocation-related sarcomas excluding myxoid liposarcoma $(n=8,40 \%)$ and others $(n=1,5 \%)$. The most common site was extremity $(n=11$, $55 \%$ ) followed by retroperitoneal ( $n=3,15 \%)$, visceral $(n=3$, $15 \%)$, and others $(n=3,15 \%)$ (-Table 1$)$.

\section{Drug Results and Outcomes}

Median number of previous lines received was 2 (range: $0-4)$. Median number of trabectedin cycles received was 4 (range: 1-17) and a total of 116 cycles were administered. Best response assessed was stable disease $(n=10,50 \%)$, progressive disease $(n=6,30 \%)$, partial response $(n=1$, $5 \%$ ), and not assessed in three patients (on treatment-2, could not be assessed-1). Five patients (25\%) had prolonged response (stable disease for $\geq 1$ year) till now. Two patients, one with gastric leiomyosarcoma and one with mesenchymal chondrosarcoma, have received 17 cycles. One patient with retroperitoneal leiomyosarcoma has received 16 cycles and another with desmoplastic small round cell tumor has received 12 cycles ( - Fig. 1 ). Out of these 4 , three patients are still on trabectedin. After a median follow-up of 19 months, median PFS was 4 months (-Table 2, -Fig. 2).

\section{Toxicity Analysis}

Seven (35\%) patients had grade 1 and 2 anemia, three patients (15\%) had grade 1 and 2 thrombocytopenia, two (10\%) patients had febrile neutropenia, $10 \%$ had rhabdomyolysis, 


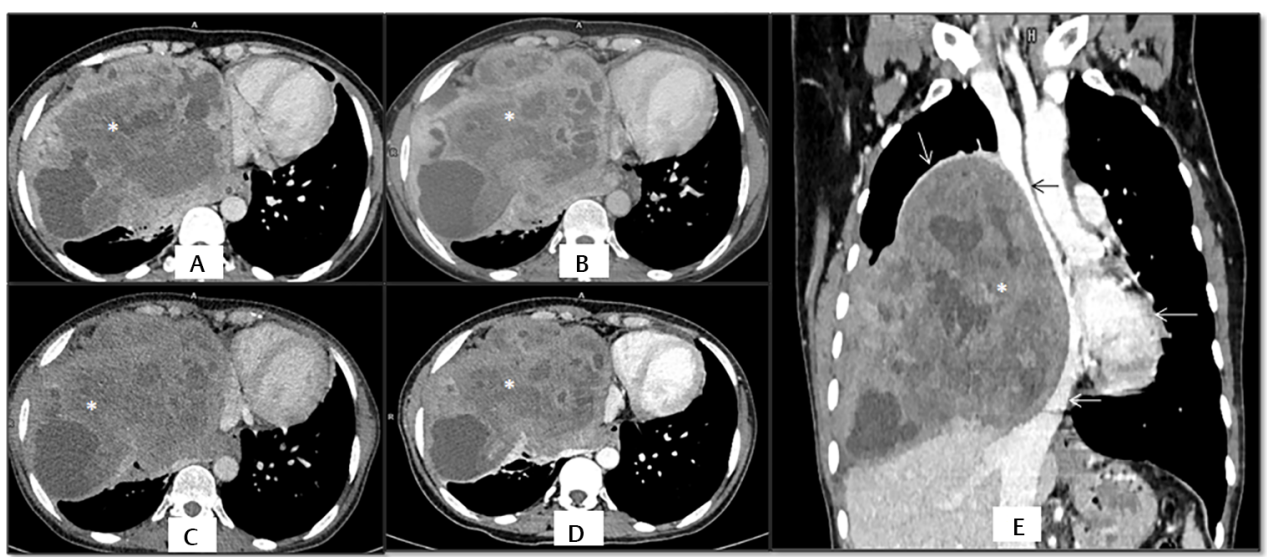

Fig. 1 Desmoplastic small round cell tumor in a female patient: Serial computed tomographic images of 2018 May (A) and December (B), 2019 May (C), and December (D) show a large heterogeneously enhancing mass (asterisk) in right hemithorax that is stable on trabectedin therapy. The mass has necrotic areas within and is causing mass effect on the mediastinal vascular structures and lung parenchyma as evident in the coronal reformatted image also (arrows in $\mathbf{E}$ ).

Table 1 Patient characteristics

\begin{tabular}{|c|c|}
\hline Characteristics $(n=20)$ & Number (\%) \\
\hline Median age (range) & $\begin{array}{l}46 \text { years }(22-73 \\
\text { years) }\end{array}$ \\
\hline \multicolumn{2}{|l|}{ Sex } \\
\hline Male & $11(55)$ \\
\hline Female & $9(45)$ \\
\hline \multicolumn{2}{|l|}{ Site } \\
\hline Extremity & $11(55)$ \\
\hline Retroperitoneal & $3(15)$ \\
\hline Visceral & $3(15)$ \\
\hline Others & $3(15)$ \\
\hline \multicolumn{2}{|l|}{ Histopathology subtype } \\
\hline Leiomyosarcoma & $8(40)$ \\
\hline Liposarcoma & $3(15)$ \\
\hline $\begin{array}{l}\text { Translocation-related sarcomas exclud- } \\
\text { ing myxoid liposarcoma }\end{array}$ & $8(40)$ \\
\hline Others & $1(5)$ \\
\hline \multicolumn{2}{|l|}{ Performance status (ECOG) } \\
\hline 0 & $0(0)$ \\
\hline 1 & $13(65)$ \\
\hline 2 & $5(0)$ \\
\hline 3 & $2(10)$ \\
\hline 4 & $0(0)$ \\
\hline
\end{tabular}

Abbreviation: ECOG, Eastern Cooperative Oncology Group.

$10 \%$ had grade 1 or 2 transaminitis and $10 \%$ had grade 1 or 2 fatigue and myalgia. No patient had grade $3 / 4$ toxicity till now.

\section{Discussion}

The problem in a resource-constraint setting with the use of trabectedin is the need of admission for 24 hours, requirement of central venous line for administration, and cost. Schöffski et al demonstrated that trabectedin could be

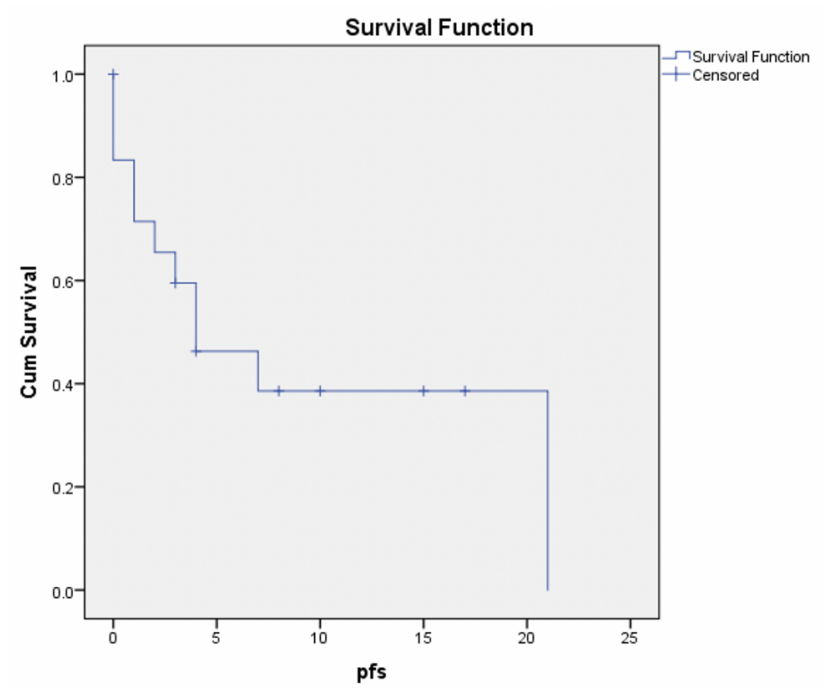

Fig. 2 Progression-free survival (PFS).

Table 2 Survival data

\begin{tabular}{|l|l|}
\hline Variable & Number \\
\hline Best response & \\
\hline Partial remission & $1(5 \%)$ \\
\hline Stable disease & $10(50 \%)$ \\
\hline Progressive disease & $6(30 \%)$ \\
\hline Not assessed & $3(15 \%)$ \\
\hline Median follow-up & 19 months \\
\hline PFS & 4 months \\
\hline
\end{tabular}

Abbreviation: PFS, progression-free survival.

administered by elastomeric pump that could be taken at home, leading to better patient satisfaction and offering the very much desired home care without compromising efficacy. ${ }^{17}$ The number of infusions by elastomeric pump till now were 16 and there were no adverse reactions associated with elastomeric infusions (-Fig. 3 ). 


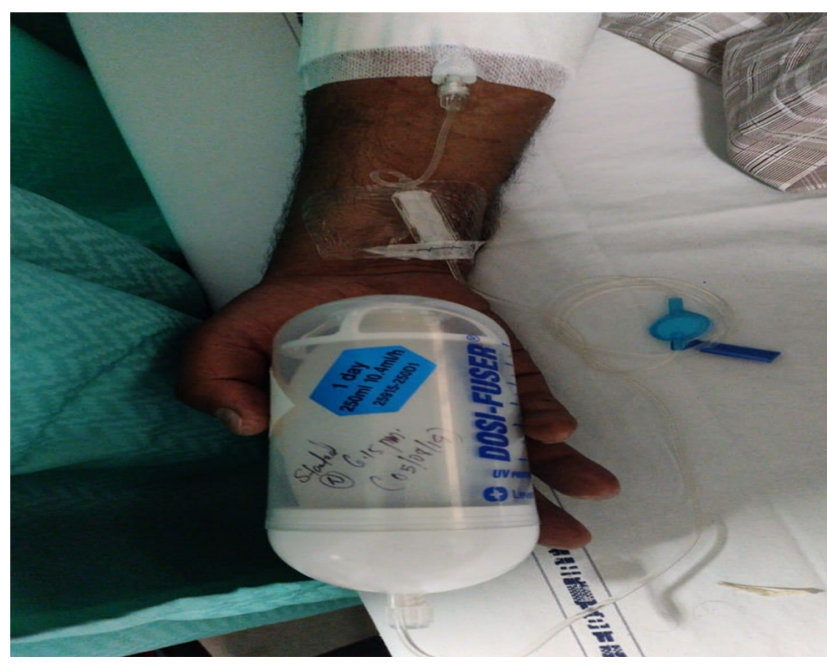

Fig. 3 Trabectedin infusion via an elastomeric pump.

Unlike other chemotherapy drugs, trabectedin is unique as it can be continued for many cycles due to lack of cumulative toxicities. ${ }^{11,18}$ One case reported a patient who had received 47 cycles of trabectedin. ${ }^{19}$ Three of our patients have received more than 15 cycles.

The median age in our patients was 46 years and most common type of sarcoma was leiomyosarcoma. Fifty-five percent of patients had L-sarcomas and $40 \%$ had translocation-related sarcomas. The median PFS was 4 months that is similar to that in literature ( 4.2 months). Of the 20 patients, 5 had prolonged stabilization, with stable disease for more than a year. The rate of clinical benefit rate in our patients was $80 \%$ compared with $60 \%$ as per literature. ${ }^{12}$

Trabectedin was well tolerated in our population. The most common adverse effects (all grades) of trabectedin in noted in trials are nausea (73\%), fatigue (67\%), neutropenia (49\%), increased ALT (alanine aminotransferase) (39\%), vomiting (44\%), anemia (39\%). etc. ${ }^{12}$ None of our patients had grade $3 / 4$ toxicities.

QoL is an important consideration when considering systemic therapies for advanced soft tissue sarcomas. ${ }^{13}$ Though the trial by Demetri et al did not report QoL, the randomized phase 3 Trial Comparing Trabectedin to the Best Supportive Care in Patients With Sarcoma (TSAR) showed that trabectedin improves PFS without impairing QoL when compared with best supportive care. ${ }^{12,20}$ Trabectedin unlike chemotherapy has no mucositis and hair loss, leading to better acceptance by patients.

Trabectedin has been tested in the setting of neoadjuvant therapy. Gronchi et al evaluated trabectedin in locally advanced myoxid liposarcoma alone and with radiotherapy (RT) in two phase 2 trials. One trial reported pathological complete response with trabectedin in 2 out of 25 patients. Thus, trabectedin is a therapeutic option in neoadjuvant setting with a favorable safety profile when used along with RT. ${ }^{21-23}$ Further trials are needed for establishing a definite role of trabectedin in neoadjuvant setting.

With prolonged therapy, drug holiday is an emerging and interesting concept. Pierantoni et al reported four cases in whom a drug holiday was given after at least 6 months of therapy with trabectedin and in three out of four patients a rechallenge at progression achieved repeat disease control or response. ${ }^{24}$ Kotecki et al reported that feasibility of such treatment breaks and rechallenge of trabectedin though continuous therapy was associated with a better PFS. ${ }^{25}$

Use of a biomarker is still eluding this drug, as in majority of sarcoma drugs, and might be a relevant research question in future.

This is a small single-center study but might be the harbinger of more data from developing countries. Elastomeric pump-based trabectedin may be preferable option given the ease of administration.

The data on trabectedin is sparse from developing countries with occasional case reports. ${ }^{26}$ There is a need of collaborative studies from the developing world.

\section{Conclusion}

Trabectedin should be considered in a setting of advanced sarcoma after first-line doxorubicin-based chemotherapy. The survival and response data trends in our experience are similar to that reported in the international literature. The advent of ambulatory infusions give an opportunity to harness the survival benefit of this drug in selected patients and at the same time giving them a better QoL. The drug is not yet approved for translocation-related sarcomas and further trials are needed in myxoid liposarcoma and translocation-related sarcoma patients. Role of immunotherapy in advanced sarcoma should also be evaluated.

\section{Conflict of Interest}

None.

\section{References}

1 Siegel RL, Miller KD, Jemal A. Cancer statistics, 2019. CA Cancer J Clin 2019;69(1):7-34

2 van der Graaf WTA, Blay J-Y, Chawla SP, et al. EORTC Soft Tissue and Bone Sarcoma GroupPALETTE study group. Pazopanib for metastatic soft-tissue sarcoma (PALETTE): a randomised, double-blind, placebo-controlled phase 3 trial. Lancet 2012;379(9829):1879-1886

3 Demetri GD, Schöffski P, Grignani G, et al. Activity of eribulin in patients with advanced liposarcoma demonstrated in a subgroup analysis from a randomized phase III study of eribulin versus dacarbazine. J Clin Oncol 2017;35(30):3433-3439

4 Zewail-Foote M, Hurley LH. Ecteinascidin 743: a minor groove alkylator that bends DNA toward the major groove. J Med Chem 1999;42(14):2493-2497

5 Allavena P, Signorelli M, Chieppa M, et al. Anti-inflammatory properties of the novel antitumor agent Yondelis (trabectedin): inhibition of macrophage differentiation and cytokine production. Cancer Res 2005;65(7):2964-2971

6 Guirouilh-Barbat J, Redon C, Pommier Y. Transcription-coupled DNA double-strand breaks are mediated via the nucleotide excision repair and the Mre11-Rad50-Nbs1 complex. Mol Biol Cell 2008;19(9):3969-3981

7 D'Incalci M, Badri N, Galmarini CM, Allavena P. Trabectedin, a drug acting on both cancer cells and the tumour microenvironment. Br J Cancer 2014;111(4):646-650 
8 Demetri GD, Chawla SP, von Mehren M, et al. Efficacy and safety of trabectedin in patients with advanced or metastatic liposarcoma or leiomyosarcoma after failure of prior anthracyclines and ifosfamide: results of a randomized phase II study of two different schedules. J Clin Oncol 2009;27(25):4188-4196

9 Monk BJ, Blessing JA, Street DG, Muller CY, Burke JJ, Hensley ML. A phase II evaluation of trabectedin in the treatment of advanced, persistent, or recurrent uterine leiomyosarcoma: a gynecologic oncology group study. Gynecol Oncol 2012;124(1):48-52

10 Yovine A, Riofrio M, Blay JY, et al. Phase II study of ecteinascidin-743 in advanced pretreated soft tissue sarcoma patients. J Clin Oncol 2004;22(5):890-899

11 Le Cesne A, Blay JY, Judson I, et al. Phase II study of ET-743 in advanced soft tissue sarcomas: a European Organisation for the Research and Treatment of Cancer (EORTC) soft tissue and bone sarcoma group trial. J Clin Oncol 2005;23(3):576-584

12 Demetri GD, von Mehren M, Jones RL, et al. Efficacy and safety of trabectedin or dacarbazine for metastatic liposarcoma or leiomyosarcoma after failure of conventional chemotherapy: results of a phase III randomized multicenter clinical trial. J Clin Oncol 2016;34(8):786-793

13 Rastogi S, Bakhshi S. Trabectedin in soft tissue sarcoma: have we hit the bull's-eye. ? J Clin Oncol 2016;34(29):3582-3583

14 Blay J-Y, Leahy MG, Nguyen BB, et al. Randomised phase III trial of trabectedin versus doxorubicin-based chemotherapy as first-line therapy in translocation-related sarcomas. Eur J Cancer 2014;50(6):1137-1147

15 Kawai A, Araki N, Sugiura H, et al. Trabectedin monotherapy after standard chemotherapy versus best supportive care in patients with advanced, translocation-related sarcoma: a randomised, open-label, phase 2 study. Lancet Oncol 2015;16(4):406-416

16 Morioka H, Takahashi S, Araki N, et al. Results of sub-analysis of a phase 2 study on trabectedin treatment for extraskeletal myxoid chondrosarcoma and mesenchymal chondrosarcoma. BMC Cancer 2016;16(1):479

17 Schöffski P, Cerbone L, Wolter P, et al. Administration of 24-h intravenous infusions of trabectedin in ambulatory patients with mesenchymal tumors via disposable elastomeric pumps: an effective and patient-friendly palliative treatment option. Onkologie 2012;35(1-2):14-17
18 Twelves C, Hoekman K, Bowman A, et al. Phase I and pharmacokinetic study of Yondelis (Ecteinascidin-743; ET-743) administered as an infusion over $1 \mathrm{~h}$ or $3 \mathrm{~h}$ every 21 days in patients with solid tumours. Eur J Cancer 2003;39(13):1842-1851

19 Konstantinos E, Christos A, Chrysanthi K, Vassiliki T, Eleni T. Complete response after long-term 2nd line treatment with trabectedin in a hemodialyzed patient with metastatic high-grade ovarian sarcoma, case report and short review. Arch Clin Med Case Rep 2019;3(6):591-599

20 Le Cesne A, Kapso Kapnang R, Foulon S, Bonastre J. 1604PDHealth-related quality of life in patients with advanced soft tissue sarcoma (ASTS): results from the TSAR randomized phase III trial of the French Sarcoma Group. Ann Oncol 2018;29(suppl_8) Available from: https://academic.oup.com/ annonc/article/29/suppl_8/mdy299.003/5142270 Accessed July 19, 2021

21 Gronchi A, Bui BN, Bonvalot S, et al. Phase II clinical trial of neoadjuvant trabectedin in patients with advanced localized myxoid liposarcoma. Ann Oncol 2012;23(3):771-776

22 Gronchi A, Hindi N, Cruz J, et al. Trabectedin and RAdiotherapy in Soft Tissue Sarcoma (TRASTS): Results of a Phase I Study in Myxoid Liposarcoma from Spanish (GEIS), Italian (ISG), French (FSG) Sarcoma Groups. EClinicalMedicine 2019;9:35-43

23 Gronchi A, Le Cesne A, Bui NB, et al. A phase II clinical trial of neoadjuvant trabectedin in patients with nonmetastatic advanced myxoid/round cell liposarcoma (MRCL. J Clin Oncol 2009;27(15 suppl):10525-10525

24 Pierantoni F, Maruzzo M, Brunello A, et al. Trabectedin drug holiday and rechallenge in soft tissue sarcomas: report of 4 cases and literature review. Front Oncol 2019;9:553, https:// www.frontiersin.org/articles/10.3389/fonc.2019.00553/full cited2019Nov18 [Internet]

25 Kotecki N, Le Cesne A, Tresch-Bruneel E, et al. Impact of trabectedin interruption and subsequent rechallenge on progression in patients with advanced soft tissue sarcoma: long-term follow-up of the T-DIS trial. Am J Clin Oncol 2018;41(11):1094-1100

26 Rastogi S, Kalra K, Manasa P, Rajawat M, Mehta V. Long lasting response of trabectedin in patient with gastric leiomyosarcoma with liver metastasis: an update to previous report. Future Sci OA 2019;6(1):FSO432 